\title{
PARAMETRES PHENOTYPIQUES ET GENETIQUES DE LA REPRODUCTION DE LA BREBIS OULED-DJELLAL (ALGÉRIE)
}

\author{
PHENOTYPIC AND GENETIC PARAMETERS FOR EWE REPRODUCTIVE \\ PERFORMANCES OF OULED-DJELLAL BREED (ALGERIA)
}

\author{
Dekhili, M. ${ }^{1 *}$ \\ 'Département d'Agronomie. Université Ferhat Abbas. Sétif 19000. Algérie. *Dekhili48@yahoo.fr
}

\section{MOTS CLÉS}

Effet environnemental. Effet génétique direct. Effet maternel. Héritabilité. Répétabilité.

\section{RÉSUMÉ}

Les performances reproductives, de 318 brebis de race Ouled-Djellal, ont été étudiées. Les paramètres génétiques ont été estimés pour cinq variables: fertilité, prolificité, taux de survie, taux de productivité numérique et taux de productivité pondéral. L'année, l'âge, le mois de saillie, la parité ont été considérés comme facteurs à effets fixés. Les effets génétiques directs et de l'environnement permanent de la brebis ont été considérés comme facteurs aléatoires. Les paramètres génétiques ont été estimés en utilisant le logiciel MTDFREML. Les héritabilités obtenues ont été faibles dans leur ensemble, ceci à cause de l'influence de l'environnement et du mode d'expression non normal de ces variables.

\section{SUMMARY}

Reproductive traits from 318 Ouled-Djellal ewes were recorded from 2008 to 2010. Genetic parameters were estimated for three basic and two composite traits. The basic traits were fertility, prolificacy and rate of survival. The composite traits were lamb weaned per ewe joined and weight of lamb per ewe joined. Year, age, parity and mating month, were considered as fixed effects. Direct genetic effects, permanent environmental effects of ewe were considered to be random effects. A derivative-free algorithm was used to obtain REML estimates of genetic environmental parameters. Estimates of heritabilities for animal genetic effects were mainly small, due to the typical high influence of environ-

\section{AdDitional KEYWORDS}

Direct genetic effect. Heritability. Maternal genetic effect. Permanent environmental effect. Repeatability.

mental factors on reproductive traits and to nonnormal distribution of traits.

\section{INTRODUCTION}

En Algérie, la race Ouled-Djellal est exploitée essentiellement pour la production de viande (Trouette, 1933; Sagne, 1950; Chellig, 1992; Dekhili, 2002). A cause de la rareté des travaux portant sur la race OuledDjellal et aussi au manque de mise en place de programmes de sélection, ses potentialités génétiques sont encore méconnues. La race Ouled-Djellal, est la race plus importante en nombre dans la région, puisque elle est arrivée à coloniser la presque totalité de la steppe, celle des hauts plateaux céréaliers de l'Algérie et une partie des pays avoisinants (Maroc et Tunisie). Beaucoup d'auteurs s'accordent à lui reconnaitre plusieurs avantages: bonnes aptitudes maternelles, de bonnes qualités de reproduction, une bonne résistance aux conditions difficiles, une bonne utilisation des aliments grossiers et de bonnes aptitudes à la marche (Trouette, 1933; Cabbée, 1959; Sagne, 1950; Chellig, 1992; Dekhili, 2002; Dekhili et Aggoun, 2005 et Mennani et al., 2011). Les travaux réalisés sur la race Ouled-Djellal sont rares, ceux qui existent ont portés sur des effectifs 
restreints et sur de courtes périodes. Selon une étude réalisée par Dekhili (2010), portant sur dix huit troupeaux, elle a permis de mesurer des paramètres zootechniques de la race dans ses conditions d'élevage habituelles, sur une longue période. Il a été difficile de porter un jugement catégorique quant aux capacités de la race. En effet, les résultats obtenus sont surtout le reflet de la conduite d'élevage, qui visiblement était médiocre. Les résultats de cette investigation ont été les suivants: le taux de survie a été de $68 \%$, la prolificité de $110 \%$, la fécondité de $93 \%$, le nombre d'agneaux sevrés par brebis luttée de $80 \%$, avec des poids des agneaux au sevrage de $12,8 \mathrm{~kg}$ seulement. Ces résultats indiquent clairement que les brebis n'étaient pas dans des conditions optimales pour pouvoir exprimer leur potentiel et permettant de pouvoir porter des conclusions. L'absence de résultats obtenus sur d'autres travaux ou d'autres races ovines Algériennes, on permett de faire une comparaison milite en faveur de cette dernière conclusion. Dekhili et Aggoun (2006a), ont évalué l'héritabilité du taux de productivité numérique de la brebis de race Ouled-Djellal, qui a été de 0,117 . Ces résultats ont suggéré l'existence d'une variabilité génétique modérée, qui pourrait répondre favorablement à la sélection. Pour faire face à l'augmentation de la population algérienne, et à la demande croissante en viande, et permettre de combler le déficit entre la demande et la production, il est nécessaire d'améliorer la productivité des troupeaux. Les variables ayant trait à la capacité reproductives de la brebis: sa capacité maternelle, sa production de lait, la croissance et la survie des agneaux, contrôlent l'efficacité de la production ovine ou des troupeaux ovins (Rao et Notter, 2000). Donc, une grande part de l'augmentation de la production des troupeaux ovins et des revenus qui seront générés, peuvent être atteints par l'amélioration des variables liées à la reproduction et la croissance des agneaux de la naissance au sevrage (Wang et Dickerson, 1991). A cet égard, la performance productive de la brebis peut être appréciée par le poids total d'agneaux produits au sevrage, qui reflète ainsi la fertilité de la brebis, le nombre d'agneaux de la naissance au sevrage.

L'objectif du présent travail consiste à l'évaluation des paramètres génétiques de caractères liés à la productivité des brebis, afin de permettre la mise en place d'un programme de sélection efficace pour l'amélioration des performances de reproduction pour les troupeaux ovins.

\section{MATÉRIELSET MÉTHODES}

Les informations individuelles des animaux utilisés dans cette étude proviennent de la ferme d'Etat Ben-Aichouche, située dans la zone céréale-élevage des hauts plateaux de l'Est Algérien. Des fiches pour chaque animal ont été mises au point pour consigner tous les événements et renseignements survenus durant les trois années de suivi (2008-2010). Chaque animal était identifié par une boucle portant un numéro, et placée au niveau de son oreille. Des boucles de couleur verte ont été attribuées aux brebis, de couleur noire aux béliers et de couleur jaune aux descendants. Le nombre de brebis étudié s'élève à 318 et 20 béliers de race Ouled-Djellal, menées en un seul lot.

\section{Conduite ALIMENTAIRE}

L'alimentation de base des animaux durant toute l'année est assurée par du foin de paille de vesce-avoine, de la paille hachée et d'herbe de pâturage en cas de besoin. Durant l'automne et le printemps, les troupeaux reçoivent de l'orge en vert et pâturent, sur de courtes durées, sur prairie de luzerne. Pendant l'été, les moutons pâturent sur chaumes de céréales d'orge et de blé tout en recevant du foin de paille. En hiver, les ovins reçoivent une complémentation d'orge en grain et du foin ou de la paille hachée. Ce mode d'alimentation est 
celui généralement pratiqué dans toute la région des Hauts-plateaux de l'Est Algérien.

\section{Conduite de la REPRODUCTION ET dE L'AGNELAGE}

Un mois avant la lutte (Mars) était pratiqué une distribution d'orge en grain à raison de $200 \mathrm{~g} / \mathrm{j} /$ par brebis et par béliers, suivi des repousses de printemps, constituant ainsi un flushing naturel. La lutte était de type contrôlée en lots, à raison de un bélier pour seize brebis (1/16). L'affectation des animaux s'est faite de manière aléatoire. La lutte a eu lieu chaque année au mois d'avril-mai: de ce fait $90 \%$ des agnelages ont eu lieu en Septembre. Tous les événements concernant l'agnelage étaient reportés dans les douze heures qui suivaient l'agnelage dans un registre (date de naissance, numéro, sexe, poids naissance, mortalité et causes, pédigrée de l'agneau etc.). Après agnelage, les agneaux et leur mère sont séparés du reste du troupeau dans des boxes aménagés a cet effet et restent avec leur mère pour une durée de deux à trois jours, ensuite ils suivent leur mère au pâturage jusque au sevrage. La désinfection du cordon ombilicale et la prise de médicament contre les septicémies ont lieu rapidement. Après cette période, les agneaux suivent leur mère (bergerie et pâturage) jusqu'au sevrage (90 jours). Les agneaux malades ou délaissés par leur mère (primipares), ont été allaités artificiellement avec du lait en poudre. D'ailleurs leur nombre est faible qu'ils ne sont pas pris en compte. Les brebis réformées dés l'âge de sept ans, par contre les béliers étaient renouvelés tous les deux ans. Aucun mode de sélection n'était utilisé dans la ferme.

\section{VARIABLES ANALYSÉES, FACTEURS TESTÉS ETMÉTHODES D'ANALYSES}

Les variables analysées sont la fertilité (Fert) ou taux d'agnelage (0 ou 1); la prolificité (Prol) ou nombre d'agneaux nés/brebis mettant bas $(1,2,3)$; le taux de survie (TS) ou nombre d'agneaux vivants au sevrage à 90 jours par brebis agnelantes $(0,1,2,3)$; le taux de productivité numériques (TPN) ou nombre d'agneaux sevrés/brebis mises à la lutte $(0,1,2,3)$; et le taux de productivité pondérale (TPP kg) ou poids d'agneaux sevrés par brebis luttée. Ces deux dernières variables constituent une mesure de la performance productive de la brebis, et sont une combinaison linéaire de plusieurs autres variables:

TPN $=$ Fert ${ }^{*}$ Prol ${ }^{*}$ TS

TPPkg $=$ Fert ${ }^{*}$ prol ${ }^{*} T S^{*}$ PSkg

oú:

$\mathrm{PSkg}=$ poids moyen des agneaux au sevrage.

Ou encore:

$T P P k g=T^{*} P S k g$

Les facteurs à effets fixés sont: l'Année (2008 à 2010); l'Age de la brebis (1 à 7 ans); la parité et le mois de saillie (Mars-Avril). Tous ces facteurs ont été testés par la méthode des moindres carrées. Les facteurs non significatifs n'ont pas été retenus dans le modèle final. Le logiciel Harvey (1990) a été utilisé pour réaliser les premières analyses par variable, afin d'obtenir le meilleur modèle en se basant sur la plus faible résiduelle.

Les seconds facteurs à effets aléatoires comprennent l'effet direct de la brebis et l'effet de l'environnement permanent de la brebis pour toutes les variables. L'estimation des paramètres génétiques a été réalisée selon le modèle animal en utilisant la méthode d'estimation des composantes de la variance par le maximum de vraisemblance restreint (REML) à l'aide du logiciel MTDFREML écrit par Boldman et al. (1993). Chaque variable a été analysée séparément pour obtenir les estimations des héritabilités et les répétabilités. Le modèle statistique pour chaque variable a été: 


\section{DEKHILI}

Modèle: $Y=X \beta+Z_{a} a+Z_{p e} p e+e$

La matrice de variance covariance des effets du modèle1 est la suivante:

$Y=$ vecteur des performances des animaux;

$\beta=$ vecteur des effets fixés (mois de saillie);

$a=$ vecteur des effets génétiques directs de la brebis;

$p e=$ vecteurs des effets environnementaux permanents de la brebis:

$\mathrm{e}=$ vecteur des résiduelles;

$\mathrm{X}, \mathrm{Z}_{\mathrm{a}}$ et $\mathrm{Z}_{\mathrm{pe}}$ constituent les matrices d'incidence.

A partir de ce modèle d'analyse, les paramètres génétiques estimés sont l'héritabilité directe $\left(\mathrm{h}^{2}\right)$, et les autres composantes qui sont $\sigma_{\mathrm{a}}^{2}$ : variance génétique additive; $\sigma_{\text {pe }}^{2}$ : variance de l'environnement permanent; $\sigma_{\mathrm{e}}^{2}$ : variance résiduelle; $\sigma_{\mathrm{p}}^{2}$ : variance phénotypique; $\mathrm{c}^{2}:\left(\sigma_{\mathrm{pe}}^{2} \sigma_{\mathrm{p}}^{2}\right)$ : proportion de la variance de l'environnement permanent par rapport à la variance phénotypique; $\mathrm{e}^{2}:\left(\sigma_{\mathrm{e}}^{2} \sigma_{\mathrm{p}}^{2}\right)$ : proportion de la variance résiduelle par rapport à la variance phénotypique (table II).

Le présent modèle utilisé, basé sur l'effet direct et de l'environnement permanent de la brebis, a été vivement suggéré par Cleot et al., 2004; Ekiz et al., 2005; Mokhtari et al., 2010.

\section{RESULTATSETDISCUSSION}

Selon les résultats de l'analyse de la variance (table I), l'année, l'âge de la brebis et la parité ont été non significatifs pour toutes les variables $(p>0,05)$. Le mois de saillie n'a été significatif que pour la fertilité $(p<0,0001)$ et non significatif pour les autres caractères. Plusieurs auteurs (Ceyland et al. (2009); Boujenane et al. (2013) ont cités l'absence d'effets significatifs de l'âge de la brebis et de l'année, alors que d'autres ont trouvé des différences significatives (Dekhili, 2004; Dekhili et Aggoun, 2005; Dekhili et Benkhlif, 2005; Dekhili et Aggoun, 2006b). Les résultats des moyennes des moindres carrées et leur erreur standard pour la fertilité, la prolificité, taux de survie, le taux de productivité numérique et le taux de productivité pondérale, figurent dans la table I. Les résultats obtenus dans cette étude sont largement supérieurs à ceux de Dekhili (2010) pour la même race. Avec une bonne conduite, non seulement des résultats encourageants peuvent être obtenus (Ekiz et al., 2005), mais l'effet de certains facteurs tels que l'année, l'âge et autres peuvent être atténués. Les coefficients de variation sont élevés ce qui peut inférer des perspectives de progrès.

Les résultats obtenus par âge, année et parité ne démontrent aucune association avec les performances de la brebis. Au contraire, la forme en dents de scie obtenue pourrait signifier la présence d'une conduite appropriée suivie avec une bonne alimentation au sein de la ferme. Cependant, les résultats obtenus lors de cette investigation sont inferieurs à ceux obtenus par Boujenane et al. (2013) concernant les brebis de race D'man.

L'estimation des composantes de la variance et des paramètres génétiques

Table I. Moyennes globales $(\mu)$, erreur standard (es), coefficient de variation $(C V, \%)$ et intervalles pour les variables analysées. (Least square means, standard errors, coefficient of variation (\%) and confidence interval for the analyzed traits)

\begin{tabular}{lccccc}
\hline & $F$ & $P$ & TS & TPN & TPP \\
\hline$\mu$ & 0,89 & 1,26 & 1,06 & 1,13 & 18,0 \\
es & 0,04 & 0,06 & 0,05 & 0,06 & 0,09 \\
CV & 38,5 & 38,7 & 43,0 & 43,8 & 41,5 \\
Intervalle & $0-1$ & $1-3$ & $0-3$ & $0-3$ & $0-40$ \\
Age & ns & ns & ns & ns & ns \\
Année & ns & ns & ns & ns & ns \\
Parité & ns & ns & ns & ns & ns \\
Mois de saillie & $* * *$ & $n s$ & $n s$ & $n s$ & $n s$
\end{tabular}

ns= non significatif; ${ }^{* * *} \mathrm{p}<0,0001$.

$F=$ fertilité; $P=$ prolificité; $T S=$ tau $x$ de survie; $T P N=$ taux productivité numérique; $\mathrm{TPP}=$ taux productivité pondérale $(\mathrm{kg})$. 
figurent dans la table II. Les héritabilités ont été de 0,$01 ; 0,06 ; 0,03 ; 0,01$ et de 0,04 pour la fertilité, la prolificité, taux de survie, taux de productivité numérique et le taux de productivité pondérale respectivement. Les estimations de paramètres obtenus sont dans l'intervalle de la large revue synthétisée par Fogarty (1995), Ekiz et al. (2005), Boujenane et al. (2013). Toutefois ces valeurs concernent d'autres races et d'autres milieux autres que ceux de l'Algérie. Les faibles estimations obtenues sont probablement dues aux effets de l'environnement aléatoire sur la variabilité des ces observations et aussi au mode d'expression ordinal de ces variables (Falconer, 1989). Le taux de productivité pondérale ou performance de la brebis, constitue une combinaison de deux variables importantes: TPN et le taux de survie (Dekhili, 2002). Le premier schématise mieux les performances reproductives de la brebis et constitue également le produit de plusieurs autres variables: Fert*Prol*TS (Dekhili, 2004). Le caractère (TPP) exprime la capacité de la brebis à produire une certaine quantité d'agneaux au sevrage après une saillie fructueuse. L'intervalle de production varie entre 0 et 40 $\mathrm{kg}$ avec un coefficient de variation de 41,5 $\%$ (tableI). La présente variable semble donc constituer un bon objectif de sélection pour améliorer la productivité totale de la brebis et de pouvoir quantifier pour une campagne d'élevage la production d'agneaux-viande.

L'estimation des variances dues à l'effet génétique ont été estimés conjointement avec les effets de l'environnement permanent de la brebis. Ces derniers ont varié de 0,0004 (Fert ) et de 1,9331 (TPP). Les premiers effets ont été importants pour la prolificité et pour le TPP et relativement faibles pour la survie et faibles pour la fertilité et le TPN. La proportion de l'effet de la variance de l'environnement permanent varie entre 0,003 à 0,59 (table II). Les estimations des répetabilités obtenues pour la fertilité, la prolificité et le taux de survie ne sont pas différentes de leurs héritabilités correspondantes. Par contre, pour les autres variables, ou les valeurs de cette fraction sont plus importantes, les valeurs de leurs correspondantes répétabilités sont plus

Table II. Estimation des composantes de la variance et des paramètres génétiques pour la fertilité (Fert), prolificité (Prol), taux de survie (TS), productivité numérique (TPN) et productivité pondérale (TPP). (Estimates of component of variance and genetic parameters for fertility, prolificacy survival, rate of numerical productivity and weight of productivity).

\begin{tabular}{lccccc}
\hline Paramètres & Fert & Prol & TS & TPN & TPP \\
\hline$\sigma^{2}$ & 0,0004 & 0,055 & 0,006 & 0,00135 & 1,93307 \\
$\sigma^{2}$ & 0,000104 & 0,01562 & 0,012 & 0,0996 & 28,6527 \\
$\sigma^{2}$ & 0,234 & 0,7443 & 0,200 & 0,06845 & 8,2362 \\
$\sigma^{2}$ & 0,03242 & 0,9659 & 0,21 & 0,2437 & 48,564 \\
$\mathrm{~h}^{2}$ & $0,01(0,04)$ & $0,06(0,02)$ & $0,03(0,04)$ & $0,01(0,01)$ & $0,04(0,005)$ \\
$r$ & 0,05 & 0,06 & 0,09 & 0,4 & 0,6 \\
$\mathrm{c}^{2}$ & 0,003 & 0,02 & 0,06 & 0,40 & 0,59 \\
$\mathrm{e}^{2}$ & 0,72 & 0,77 & 0,95 & 0,28 & 0,17 \\
\hline
\end{tabular}

$\sigma_{\mathrm{a}}^{2}=$ variance génétique additive; $\sigma_{\mathrm{pe}}^{2}=$ variance de l'environnement permanent; $\sigma_{\mathrm{e}}^{2}=$ variance résiduelle; $\sigma_{p}^{2}=$ variance phénotypique; $h^{2}=\left(\sigma_{a}^{2} \sigma_{p}^{2}\right)=$ héritabilité et leur erreur standard; $r=\left(\sigma_{a+}^{2} \sigma_{p e /}^{2} \sigma_{p}^{2}\right)$ repetabilité; $\mathrm{C}^{2}=\left(\sigma_{\mathrm{pe}}^{2} \sigma_{\mathrm{p}}^{2}\right)=$ proportion de la variance de l'environnement permanent par rapport à la variance phénotypique; $\mathrm{e}^{2}=\left(\sigma_{\mathrm{e},}^{2} \sigma_{\mathrm{p}}^{2}\right)=$ proportion de la variance résiduelle par rapport à la variance phénotypique. 
élevées, qui varient entre 0,4 (TPN) et 0,6 (TPP). En plus du mode d'expression et de l'influence de facteurs non considérés dans cette étude, l'environnement pourrait être la principale cause de ces faibles valeurs. On peut voir que l'influence de l'environnement est très importante pour la fertilité (72 \%), prolificité (77\%) et $95 \%$ pour le TS, par contre on constate une faible influence le taux de productivité numérique $(28 \%)$ et de $17 \%$ pour le TPP. Cette influence était prévisible, car il s'agit de l'étude de variables ou l'environnement joue un rôle fondamental sur l'efficacité de la performance de la brebis. Cependant, la réponse biologique de la race Ouled-Djellal s'inscrit bien dans les intervalles des valeurs citées de la bibliographie par beaucoup d'auteurs. Dans leur ensemble les héritabilités sont faibles, l'amélioration des variables de la reproduction de la brebis, serait difficile à réaliser, quoiqu'elles aient une grande importance économique. Pour remédier à cela, plusieurs auteurs suggèrent l'utilisation de la sélection indirecte comme moyen à utiliser (Rosati et al., 2002; Boujenane et al., 2013). Ce qui nécessite une seconde investigation basée sur l'évaluation des différentes corrélations entre plusieurs variables.

\section{CONCLUSION}

Des estimations de variances génétiques, de l'environnement permanent de la brebis et des héritabilités ont été obtenues pour la première fois concernant la OuledDjellal, dans le but d'une évaluation génétique pour la mise au point d'un programme de sélection. Les variables analysées, fertilité, prolificité, taux de survie, taux de productivité numérique et taux de productivité pondéral, sont économiquement importantes quoique manifestant des variances génétiques faibles, cependant elles doivent impérativement être considérées dans tout programme d'amélioration.
La variabilité du taux de productivité pondéral, constitue un caractère dit composé est fortement influencé par d'autres variables, telles que la fertilité, la prolificité et le taux de sevrage. Dans un milieu défavorable, la variabilité des ces caractères peut avoir une influence néfaste sur le taux de productivité pondéral. Au contraire, lorsque la conduite est favorable, la variabilité de Fert, Prol et de TS est atténuée sur TPP. Donc, l'amélioration de la conduite devient un préalable à atteindre avant d'entreprendre tout programme d'amélioration et de sélection: alimentation, préparation de la lutte et de l'agnelage, conditions sanitaires etc. En effet, améliorer l'efficacité de la production ovine ou la performance reproductive de la brebis consiste à augmenter le nombre d'animaux disponible pour la sélection, plus de produits à réformer et à vendre, augmente l'intensité de sélection, contrairement à une performance reproductive faible. Au regard des résultats obtenus, la sélection directe basée sur la propre performance de la brebis, à de faibles chances de donner des résultats encourageants. L'amélioration des variables de la fertilité de la brebis nécessitent l'utilisation de deux voies qui sont préconisées par plusieurs auteurs. Tout programme de sélection devrait être basé sur: un, sur la propre performance de la brebis et sur la performance de femelles de la même famille ou très proche, en utilisant le BLUP méthode; Deux, Il est aussi conseiller de faire usage de la sélection indirecte ou utiliser les variables en corrélations, ayant des corrélations génétiques élevées et positives pour les traits de la productivité de la brebis.

\section{REMERCIEMENTS}

Les auteurs de ce travail remercient vivement tous les responsables de la ferme Ben-Aichouche pour leur aide dans la réalisation de cette étude. 


\section{BIBLIOGRAPHIE}

Bolman, K.G.; Kriese, L.A.; Van Vleck, L.D. and Kachman, S.D. 1993. A manual for use of MTDFREML: asset of program to obtain estimates of variances and covariances. ARS, USDA, USMARC. Clay Center, NE.

Boujenane, I.; Chikhi, A.; Sylla, M. and Ibnelbachyr, M. 2013. Estimation of genetic parameters and genetic gains for reproductive traits and body weight of D'man ewes. Small Ruminant Res, 113: 40-46.

Cabbée, M. 1959. Le mouton en Algérie. Bull Tech Inf Ing Serv Agric, $\mathrm{N}^{\circ} 42$.

Ceylan, A.; Sezenier, T. and Erdogan, I. 2009. The estimation of variance component for prolificacy and growth traits of Sakiz sheep. Livest Sci, 122: 68-72.

Chellig, R. 1992. Les races ovines algériennes. Office des Publications Universitaires. BenAknoun, Alger.

Cleot, S.; Gilmour, A.; Olivier, J. and Wyk, J. 2004. Genetic and phenotypic trends and parameters in reproduction, greasy fleece weight and liveweight in Merino lines divergently selected for multiple rearing ability. Aust J Exp Agr, 44: 745-754.

Dekhili, M. 2002. Performances reproductives des brebis Ouled-Djellal nées simples et doubles. $9^{\text {es }}$ Rencontres autour des Recherches des Ruminants. INRA, 9: 155.

Dekhili, M. 2004. Étude de la productivité d'un troupeau de brebis de race Ouled-Djellal. $11^{\text {es }}$ Rencontres autour des Recherches des Ruminants. INRA, 11: 234.

Dekhili, M. et Benkhlif, R. 2005. Bilan portant sur les performances reproductives d'un troupeau de brebis Ouled-Djella. $12^{\text {es }}$ Rencontres autour des Recherches des Ruminants. INRA, 12: 162.

Dekhili, M. et Aggoun, A. 2005. Productivité des brebis Ouled-Djellal, élevées dans deux milieux différents. $12^{\text {es }}$ Rencontres autour des Recherches des Ruminants. INRA, 12: 163.

Dekhili, M. et Aggoun, A. 2006a. Paramètres génétiques de la productivité numérique des brebis Ouled-Djellal. 13 ${ }^{\text {es }}$ Rencontres autour des Recherches des Ruminants. INRA, 13: 221

Dekhili, M. et Aggoun, A. 2006b. Productivité pon- dérale des brebis Ouled-Djellal dans la zone Tellienne (Nord) de l'Algérie. $13^{\text {es }}$ Rencontres autour des Recherches des Ruminants. INRA, 13: 391

Dekhili, M. 2010. Fertilité des élevages ovins type «Hodna» menés en extensif dans la région de Sétif. Agronomie, № 0 : 1-7.

Ekiz, B.; Ozcan, M. and Yilmaz, A. 2005. Estimates of phenotypic and genetic parameters for ewe productivity traits of Turkish Merino (Karacabey Merino) sheep. Turk J Anim Sci, 29: 557-564.

Falconer, D.S. 1989. Introduction to quantitative genetics. $3^{\text {rd }}$ ed. Wiley. New-York.

Fogarty, N.M. 1995. Genetic parameters for live weight, fat and muscle measurements, wool production and reproduction in sheep: a review. Anim Breed Abstr, 63: 101-143.

Harvey, W.R. 1990. LSMLMW and MIXMDL. PC-2. Version1-21. User's Guide. Ohio State Univ. Columbus.

Mokhtari, M.; Rashidi, A. and Esmailizadeh, A. 2010. Estimates of phenotypic and genetic parameters for reproductive traits in Kermani sheep. Small Ruminant Res, 88: 27-37.

Mennani, A.; Dekhili, M. et Khlifi Ahmed, A.E. 2011. Effet du mode de lutte sur la productivité des brebis de race Ouled-Djellal. 15 ${ }^{\text {es }}$ Rencontres autour des Recherches des Ruminants. INRA, 18: 102.

Rosati, A.; Moussa, E.; Van Vleck, L. and Youg, L. 2002. Genetic parameters of reproductive traits in sheep. Small Ruminant Res, 43: 65-74.

Rao, S. and Notter, D.R. 2000. Genetic analysis of litter size in targhee Suffolk, and Polypay sheep. J Anim Sci, 78: 2113-2120.

Sagne, J. 1950. L'Algérie pastorale. Ses origines, sa formation, son passé, son présent, son avenir. Imprimerie Fontana. Alger. 27 pp.

Trouette, G. 1933. La sélection ovine dans le troupeau indigène. Direction des services de l'élevage. Imprimerie P. Guiauchin. Alger. 1-10.

Wang, C.T. and Dickerson, G.E. 1991. Simulated effects of reductive performance on life-cycle efficiency of lamb and wool production at three lambing intervals. J Anim Sci, 69: 4338-4347.

Archivos de zootecnia vol. 63, núm. 242, p. 275. 